\title{
Atividade de monitoria em Atenção Secundária em Saúde Bucal em tempos de pandemia: um relato de experiência
}

\author{
Monitoring activities in Secondary Care in Oral Health in pandemic times: an experience report \\ Importancia del seguimiento en Atención Secundaria en Salud Bucal en medio pandemia de Covid- \\ 19: informe de experiencia
}

\section{Resumo}

Este é um estudo descritivo do tipo relato de experiência acerca das atividades de educação em saúde desenvolvidas na monitoria da disciplina de Atenção Secundária em Saúde Bucal I, do curso de Odontologia da Universidade de Pernambuco (UPE), durante a pandemia do novo coronavírus. O objetivo do presente trabalho é apresentar um relato de experiência das atividades desenvolvidas na monitoria de Atenção Secundária em Saúde Bucal I que assistem os alunos do sexto período do curso de odontologia da UPE/Campus Arcoverde durante a pandemia da Covid-19. Foi utilizada como estratégia de busca e sistematização dos artigos, o levantamento de estudos indexados nas bases de dados: Scielo, Lillacs, Pubmed e Google Acadêmico, a partir dos seguintes descritores: Educação em Odontologia; Ensino; Educação à Distância; Odontologia; Covid19. A pesquisa abrangeu artigos entre o período de 2018 a 2020 , sem restrição de idioma. As atividades de monitoria desenvolvidas durante a disciplina proporcionaram experiências e conhecimentos mútuos durante todos os encontros, desde as aulas remotas acompanhadas até as práticas laboratoriais. A monitoria nessa nova realidade permitiu adaptação à realidade de ensino remoto, fortaleceu o trabalho em equipe e possibilitou experiência prática ao longo da execução das atividades nos laboratórios.

Palavras-chave:Educação em odontologia; Ensino; Educação à distância; Covid-19.

\begin{abstract}
This is a descriptive study of the experience report type about the activities of health education developed in the monitoring of the discipline of Secondary Care in Oral Health I, of the Dentistry course at the University of Pernambuco (UPE), during the new coronavirus pandemic. The objective of this paper is to present an experience report of the activities developed in the monitoring of Secondary Care in Oral Health I that assist students in the sixth period of the dentistry course at UPE/Campus Arcoverde during the Covid-19 pandemic. As a search strategy and systematization of articles, the survey of studies indexed in the following databases was used: Scielo, Lillacs, Pubmed and Academic Google, based on the following descriptors: Education in Dentistry; Teaching; Distance Education; Dentistry; Covid19. The search covered articles between the period 2018 to 2020, with no language restrictions. The monitoring activities developed during the course provided mutual experiences and knowledge during all meetings, from remotely monitored classes to laboratory practices. Monitoring in this new reality allowed for adaptation to the reality of remote learning, strengthened teamwork and enabled practical experience throughout the execution of activities in the laboratories.
\end{abstract}

Keywords: Education in dentistry; Mentors; Distance education; Covid-19.

\section{Resumen}

Se trata de un estudio descriptivo del tipo relato de experiencia sobre las actividades de educación sanitaria desarrolladas en el seguimiento de la disciplina de Atención Secundaria en Salud Bucal I, del curso de Odontología de la Universidad 
de Pernambuco (UPE), durante la nueva pandemia de coronavirus. El objetivo de este trabajo es presentar un relato de experiencia de las actividades desarrolladas en el seguimiento de la Atención Secundaria en Salud Bucal I que asisten a los estudiantes del sexto período de la carrera de Odontología en la UPE / Campus Arcoverde durante la pandemia de Covid-19. Como estrategia de búsqueda y sistematización de artículos se utilizó la encuesta de estudios indexados en las siguientes bases de datos: Scielo, Lillacs, Pubmed y Academic Google. La búsqueda abarcó artículos entre el período 2018 a 2020, sin restricciones de idioma. El seguimiento en esta nueva realidad permitió la adaptación a la realidad del aprendizaje a distancia, fortaleció el trabajo en equipo y posibilitó la experiencia práctica a lo largo de la ejecución de las actividades en los laboratorios.

Palabras clave: Educación en odontología; Mentores; Educación a distancia; Covid-19.

\section{Introdução}

O componente curricular de Atenção Secundária em Saúde Bucal I tem caráter multidisciplinar que contempla uma carga horária de 90 horas teórico-práticas, vivenciada pelos alunos do sexto período. O suporte aos alunos é feito de forma individualizada e coletiva, usando, como ferramentas metodológicas, simulados e estudos de caso, a fim de enriquecer e instigar o conhecimento dos mesmos. A atividade de monitoria, incorporada no Brasil por meio da lei federal $\mathrm{n}^{\mathrm{o}} 5.540$, de 28 de novembro de 1968, é um processo pelo qual (DM) auxiliam seus colegas de curso em processos de ensino-aprendizagem, sob orientação do docente responsável pela disciplina (Azevedo et al., 2020). Desta forma, a monitoria pode ser definida como uma estratégia de melhoria do ensino de graduação, através da utilização de práticas e experiências pedagógicas que incluem a consolidação entre aspectos teóricos e práticos, além do reconhecimento do docente perante as dificuldades que ainda encontradas após a realização das atividades na sala de aula (Vicenzi et al., 2016). A monitoria dá suporte às aulas práticas e, por vezes, auxilia nas didáticas junto ao professor (Matoso, 2014).

As atividades desenvolvidas no decorrer da monitoria acadêmica proporcionam autonomia e autoconfiança do DM por meio de aumento do contato com a disciplina e vínculo com o docente (Oliveira, et al., 2019). Frente à pandemia do novo coronavírus (Covid-19), que se institui um dos mais relevantes problemas de saúde pública na realidade mundial do século 21, o ensino presencial nas universidades acabou sendo suspenso, com o intuito de diminuir a transmissão do vírus, conforme a Declaração de Emergência em Saúde Pública emitida pela Organização Mundial da Saúde em 30 de janeiro de 2020 e da Lei $\mathrm{n}^{\circ} 13.979$, de 6 de fevereiro de 2020, que dispõe sobre as medidas de enfrentamento à Covid-19, abrangendo o isolamento social e a quarentena. O surto do vírus da Covid-19, que se alastrou rapidamente por diversas regiões do mundo, colocou os países em situação de emergência, trazendo uma diversidade de impactos, afetando principalmente as esferas da economia e educação (Spinelli \& Pellino, 2020). A disseminação da doença por Covid-19 surgida em dezembro de 2019, já alcançou os critérios epidemiológicos declarados como pandemia, em comparação com o cenário mundial de retrocesso do coronavírus, o Brasil caminhou para o crescimento de casos sem jamais ter saído de patamares altos de óbitos e casos (Pinto, 2021).

Neste momento em que todas as ações estão voltadas à proteção dos indivíduos e coletividade, com medidas de isolamento social sendo adotadas, infere-se que a pandemia no Brasil afetou de forma abrupta o processo de ensino e aprendizagem, em consequência da paralisação temporária das universidades, incluindo não somente atividades de ensino, mas também de extensão e pesquisa. Nesse sentido, a maioria das instituições de ensino foi em busca de soluções que diminuíssem os efeitos do isolamento na rotina dos discentes, e uma das estratégias encontradas foi o uso das Tecnologias da Informação e da Comunicação virtuais, com o objetivo de continuar as atividades a distância, como a de ensino (Cunha \& Bizelli, 2016). O ano de 2020 foi marcado por mudanças e adaptações consecutivas. O fechamento da universidade mudou completamente a didática e o cronograma definido na disciplina. Formas alternativas e estratégias emergenciais temporárias tiveram que ser estipuladas para que o processo de ensino-aprendizagem tivesse continuidade. No entanto, foi necessário instituir aulas teóricas de forma remota com dias alternados para as práticas com a turma dividida em datas pré-determinadas (Moreira et al., 2020). O objetivo do trabalho é apresentar um relato de experiência das atividades desenvolvidas na monitoria de Atenção Secundária em Saúde 
Bucal em 2020, que assiste os alunos do sexto período do curso de odontologia da UPE campus Arcoverde durante a pandemia do Covid-19.

\section{Metodologia}

Trata-se de um estudo descritivo, que objetiva relatar a experiência de monitoria realizada a partir da vivência em relação às atividades desenvolvidas na disciplina de Atenção Secundária em Saúde Bucal em meio à pandemia do Covid-19, no período referente ao semestre 2020.1. Também foi realizada uma pesquisa bibliográfica, onde foi utilizado como estratégia de busca e sistematização dos artigos, o levantamento de estudos indexados nas bases de dados: Scielo, Lillacs, Pubmed e Google Acadêmico. A pesquisa abrangeu artigos entre o período de 2018 a 2020, sem restrição de idioma. A estratégia de pesquisa combinou os seguintes descritores: Educação em Odontologia; Ensino; Educação à Distância; Odontologia; Covid19. A fim de delimitar os artigos que se adequam ao título do estudo, foram descartados estudos que não tinham como enfoque o tema pesquisado.

Quanto ao número de artigos encontrados em cada base de dados, a partir da combinação dos descritores supracitados 04 foram da base de dados Scielo, 63 Lillacs, 01 Pubmed, e 93 Google Acadêmico, sendo 161 artigos encontrados no total. Após leitura de títulos 88 artigos foram descartados, 04 artigos fugiram do tema, 73 resumos lidos. Quantos atribuídos a partir do resumo para leitura do texto completo 11 . Artigos citados no trabalho 03.

\section{Resultados}

As atividades de monitoria desenvolvidas durante a disciplina de Atenção Secundária em Saúde Bucal I proporcionaram experiências e conhecimentos mútuos durante todos os encontros, desde as aulas remotas acompanhadas até as práticas laboratoriais. Em meio ao contexto atual de pandemia, o planejamento da disciplina teve que se adequar às normas de segurança e distanciamento social para acontecer. Ficou sob responsabilidade da monitoria acompanhar as aulas em ambiente virtual e as atividades em laboratório; preparar materiais didáticos a serem utilizados como instrumento de estudo e revisão; elaborar e organizar atividades que contribuíssem para o conhecimento dos discentes; estar ao lado do discente para sanar suas dúvidas sobre os conteúdos teóricos e práticos oferecidos na disciplina, de forma a facilitar o processo ensino-aprendizagem, bem como auxiliá-los na correção das atividades propostas.

A experiência vivenciada durante a monitoria despertou um olhar mais profundo sobre as especialidades oferecidas na disciplina em meio à nova realidade do Covid-19, proporcionando segurança e confiança sobre determinados assuntos. O laboratório apresentou casos clínicos que na prática poderão ocorrer na clínica ou no dia a dia, tornando o monitor apto a solucionar, resultando em autoconfiança que a realidade exige. Assim, a monitoria permitiu contato direto com as dificuldades, desafios, problemas e resultou no melhor preparo para enfrentar o seu campo de trabalho.

No âmbito da colaboração universitária contribuiu substancialmente para a formação acadêmica, enriqueceu o currículo, e auxiliou nas aprendizagens dos alunos monitorados, principalmente, em relação ao compartilhar de experiências e conhecimentos. Além de estimular a ampliar a visão do conhecimento específico da área de formação e estimular no desenvolvimento da responsabilidade, incentivo a docência superior, comprometimento com a profissão, seriedade e segurança, posturas essenciais na vida profissional.

Dentre as atividades acompanhadas pelo monitor, a de maior planejamento e poder estruturante foi o acompanhamento das atividades no laboratório das especialidades cirurgia, periodontia e endodontia para atuação na atenção secundária em saúde bucal, detalhadas na Tabela 1. Teve como objetivo melhor desenvolver as aulas práticas; as demonstrações e execuções eram praticadas em sala, com ênfase na promoção de saúde e no tratamento de forma multidisciplinar, interdisciplinar e integrada, contemplando a formação generalista, humanista, crítica e reflexiva do Cirurgião-Dentista. Além disso, diversas atividades 
teóricas foram desenvolvidas, buscando não somente a resolução, mas o levantamento de discussões construtivas sobre determinados assuntos.

Tabela 1 - Detalhamento das atividades desenvolvidas.

\begin{tabular}{|c|c|c|}
\hline Atividades Desenvolvidas & Dificuldades & Superações \\
\hline $\begin{array}{l}\text { Diagnóstico e tratamento de patologias } \\
\text { endodônticas. }\end{array}$ & $\begin{array}{l}\text { Instrumentação de pré-molar superior de } \\
2 \text { canais; } \\
\text { Realização do retratamento; }\end{array}$ & $\begin{array}{l}\text { Tratamento de canal com todas as etapas } \\
\text { do preparo químico-mecânico a } \\
\text { obturação bem executados por todos os } \\
\text { alunos; } \\
\text { Meta alcançada de todos os dentes } \\
\text { propostos; }\end{array}$ \\
\hline $\begin{array}{l}\text { Técnicas cirúrgicas de exodontia de } \\
\text { dentes inclusos }\end{array}$ & $\begin{array}{l}\text { A anatomia radicular divergente de cada } \\
\text { dente; } \\
\text { Dificuldade de encontrar o dente incluso; }\end{array}$ & $\begin{array}{l}\text { Execução da técnica realizada por todos } \\
\text { os alunos. }\end{array}$ \\
\hline $\begin{array}{l}\text { Diagnóstico e tratamento de traumas } \\
\text { dentários. }\end{array}$ & $\begin{array}{l}\text { Classificação, Características, } \\
\text { Condutas corretas para cada fratura. }\end{array}$ & $\begin{array}{l}\text { Determinação do diagnóstico inicial } \\
\text { correto, e desenvolver um plano de } \\
\text { tratamento eficaz }\end{array}$ \\
\hline $\begin{array}{l}\text { Técnicas cirúrgicas de Plastias } \\
\text { Mucogengivais }\end{array}$ & Execução correta das suturas. & $\begin{array}{l}\text { Passado todo o referencial teórico e aula } \\
\text { prática demonstrativa em laboratório } \\
\text { realizada através da mandíbula de porco, } \\
\text { a turma teve sucesso praticando todas as } \\
\text { técnicas apresentadas. }\end{array}$ \\
\hline
\end{tabular}

Fonte: Brito (2021)

O auxílio que o monitor proporcionou ao docente durante as aulas, facilitou em termos de otimização do tempo no desenvolvimento das atividades. A atenção aos discentes para sanar dúvidas, resultou em um melhor aproveitamento do componente curricular. Para um melhor aproveitamento deste, várias metodologias de ensino foram desenvolvidas. Devido ao início da pandemia da Covid-19, as atividades presenciais da monitoria tiveram que ser adaptadas ao ambiente virtual, onde semanalmente os alunos tiveram as aulas teóricas através da plataforma de reuniões Google Meet, e o suporte prático no laboratório aconteceu devido à divisão das turmas e horários para segurança de todos; além disso todo o material didático com todos os conteúdos, era disponibilizado através de uma plataforma virtual, com a disponibilidade dos monitores para tirar dúvidas quando solicitado pela turma.

A incorporação das didáticas no ambiente virtual para fins educacionais mostrou-se vantajosa, pois colaborou com o desenvolvimento da autonomia do monitor em dominar os conteúdos e gerou interatividade com os conteúdos diários com monitor e docente. Em decorrência da flexibilidade e estratégias articuladas no tempo encontrado na pandemia, a facilidade de adaptação criou infinitas possibilidades de recursos para incrementar o ensino prático, possibilitando o desenvolvimento da disciplina.

Apesar de toda essa experiência desafiadora vivenciada na disciplina durante a pandemia, na qual as ferramentas tecnológicas forneceram um suporte essencial possibilitando o acompanhamento dos alunos e o desenvolvimento satisfatório das atividades, com o objetivo de avaliar a aplicabilidade destes recursos durante a monitoria, foi desenvolvido e disponibilizado um questionário aos alunos para ser respondido de forma anônima e voluntária. Desta forma, exemplifica-se a importância de adotar formas alternativas de comunicação para promover a interação entre monitores e alunos, viabilizando os objetivos da atividade de monitoria acadêmica. 


\section{Discussão}

A pandemia da Covid-19 trouxe, dentre as medidas em saúde pública adotadas pelos governos centrais de todos os países, o isolamento social obrigatório como uma das principais medidas, em virtude do crescimento exponencial das infecções pela nova doença. Nessa realidade, era inevitável o atraso ou até mesmo a paralisação das aulas em diferentes universidades do mundo. Frente a problemática em meio à crise, a educação virtual surge como uma possibilidade indispensável para dar continuidade a metodologia de ensino e formação universitária mediante ao cumprimento das medidas de isolamento e distanciamento social (Faria, et al., 2020). Em concordância, o estudo de Faria et al. (2020) afirma que o uso de ferramentas virtuais reinventou as estratégias de comunicação em saúde, favorecendo a articulação entre discentes e docentes, proporcionando a disseminação de conteúdos educativos em saúde em meio ao enfrentamento da Covid-19. Rui e Dávila (2016) citam, dentre os diversos benefícios de um processo ensino-aprendizagem virtual: amplo acesso à navegação na Internet; diversidade de formas de organização do ensino (videoconferências, fóruns, consultas, seminários, entre outros); independência, autonomia e protagonismo dos alunos, como a diversidade de ofertas e inovação que se especifica na alta eficiência dos processos educacionais.

A pandemia do coronavírus paralisou as atividades presenciais de $91 \%$ dos estudantes no mundo (United Nations Educational, 2020). O fechamento das instituições ocorreu resultante das projeções realizadas a partir de dados científicos, nas quais se considerava que o período de isolamento perduraria por, no máximo, 90 dias (World Health Organization, 2020) Devido à necessidade de adaptação à nova realidade, os países tomaram algumas atitudes: nos países da União Europeia, as instituições de ensino foram fechadas ou foi evitado por meio de alterações no calendário acadêmico como feito na Alemanha (World Health Organization, 2020); no Reino Unido e Austrália, escolas ficaram abertas para filhos de trabalhadores em setores essenciais e para crianças em situação de vulnerabilidade (Motiejünaitê-Schulmeister e Crosier, 2020); na China, houve um acréscimo de acesso à Internet e oferta de disciplinas ou cursos por meio de plataformas geridas por instituições de ensino (Government Of United Kingdom 2020). Ressalta-se, ainda, que países como Portugal, Inglaterra, Espanha e França, que elaboraram estratégias de ensino remoto, apresentaram algumas dificuldades na implementação da educação devido a diversos fatores, como, as dificuldades apresentadas por docentes e discentes em acompanhar as aulas e a falta de acesso da população às ferramentas e tecnologias de comunicação e informação, mesmo que esses países possuíssem políticas para o acesso a equipamentos eletrônicos (The World Bank 2020).

Entretanto, as projeções científicas revelaram a necessidade de aumentar os períodos de quarentena, ainda que de maneira intermitente, de forma que o retorno às atividades presenciais ocorresse de forma controlada, para que minimize os riscos de contágio (Arruda, 2020). Universidades, departamentos acadêmicos e cursos universitários precisaram se adaptar para minimizar prejuízos pedagógicos e riscos à saúde pública, para que assim fosse possível assegurar a manutenção de uma educação em nível superior de qualidade e segura. Os ajustes precisaram ser feitos nos planos de desenvolvimento da instituição, nos projetos pedagógicos de cursos a fim de enfrentar a situação de emergência. O atual contexto de pandemia da Covid-19 exigiu das Instituições de Ensino decisões sobre como encarar os processos de ensinar e aprender de modo que as partes envolvidas (professores, estudantes e funcionários) se protegessem da contaminação e da disseminação do vírus (Hodges, 2020).

As respostas que as Universidades estão construindo às demandas sociais e de ciência, tecnologia e inovação à Pandemia da Covid-19, revelam características de sua governança, adaptabilidade, otimização de recursos e de ativação rápida de recursos humanos e capacidade de pesquisa. A complexidade da diversificação, do alcance e do ritmo próprio de cada uma delas, torna difícil a compreensão e a análise sobre a forma com que estão respondendo à Pandemia (Castioni \& Melo, 2020). Corroborando com a afirmação da importância dos deveres da monitoria, Cardoso e Araújo (2008) retrataram em suas pesquisas algumas contribuições da monitoria para a formação acadêmica e enquanto futuro profissional: atuação dos monitores enquanto facilitadores do processo de ensino-aprendizagem; estímulo à docência; participação na construção de conhecimento e prática 
da monitoria como um adicional curricular, uma vez que o aluno monitor pode atuar diretamente no auxílio da formação em enfermagem, sendo, portanto um agente definidor de mudanças.

De todo modo, o ensino híbrido apresenta vantagens e pontos fracos quando empregado no ensino da Odontologia. As vantagens relatadas incluem menor tempo de deslocamento, permitindo mais tempo em casa; implantação de novas tecnologias; acesso a congressos mundiais; possibilidade de maior participação dos alunos. Atribui-se este último fenômeno, à possibilidade de interação mais direta por meio de mecanismos como os chats das plataformas. Em contrapartida, a maior parte dos alunos relatam cansaço com o uso excessivo de telas, sendo a falta de contato humano outro grande inconveniente (Cardoso \& Araújo, 2008).

O ensino híbrido é uma nova forma de ressignificar os processos de ensino e aprendizagem numa ambiência educacional que está imersa na Cultura Digital (Costa, 2021). Por esse motivo, pode ser considerada como disruptiva, pretendendo valorizar o melhor do presencial e do remoto. Pode ser considerado como uma grande aposta para o processo de ensino e aprendizagem no século XXI, devido ao seu modelo que une as melhores práticas das modalidades à distância e presencial, com potencialidades capazes de gerar uma revolução na forma de ensino e aprendizado de instituições de ensino superior (Oliveira, et al., 2021).

Conforme citado por Tokuç e Varol (2020) em estudo acerca da educação médica na Turquia durante a pandemia, a epidemia do coronavírus demonstrou um cenário de completa imprevisibilidade, gerando marcantes efeitos que podem provocar sérias mudanças de como os profissionais do futuro serão formados. Desse modo, verifica-se que o formato de aulas on-line possivelmente poderá integrar o currículo acadêmico por um prazo indeterminado, corroborando a relevância de atividades como as demonstradas neste estudo.

Deste modo, a monitoria, mesmo que em parte de maneira virtual, se revela como uma ferramenta eficiente de intervenção em meio ao processo de ensino-aprendizagem, fato este confirmado em outros estudos, nos quais demonstraram que a monitoria é uma das intervenções pedagógicas mais úteis no meio acadêmico, ao diminuir, o tempo necessário para adquirir conhecimentos considerados elementares (Frison, 2016). Além de ser uma aliada no auxílio dos acadêmicos de forma satisfatória, os ganhos dos monitores vão muito além de títulos, pois dizem respeito a ganhos pessoais e intelectuais proporcionados pela troca de conhecimento entre todos os acadêmicos e o corpo docente (Lins, et al., 2009).

\section{Conclusão}

A estratégia adotada possibilitou aos monitores maior estímulo ao estudo, devido à necessidade de elaboração dos materiais para postagem. Além de melhoria da capacidade de organização, visto que havia uma carga horária e atividades a serem cumpridas. Estes comportamentos acarretam um maior amadurecimento da autonomia. A monitoria nessa nova realidade permitiu adaptação à realidade de ensino remoto, fortaleceu o trabalho em equipe e possibilitou experiência prática ao longo da execução das atividades nos laboratórios.

\section{Referências}

Arruda, E. P. (2020). Educação Remota Emergencial: elementos para políticas públicas na educação brasileira em tempos de Covid-19. Revista de Educação a Distância, 7 (1), 257-27.

Azevedo, C. R. L, Farias, M. D. L., \& Bezerra, C. C. (2020). Monitoria acadêmica em uma disciplina semipresencial: relato de experiência. Research, Society and Development, 9 (4), 1-9. http://dx.doi.org/10.33448/rsd-v9i4.2788

Cardoso, M. V. \& Araújo, R. P. (2008). Monitoria acadêmica: relato de experiência em disciplina aplicada a terapia ocupacional. Cadernos de terapia ocupacional da UFSCar, 16 (1), 53-57.

Castioni, R., \& Melo A. A. S. (2020). Universidades do Brasil, da Espanha e da Itália na Pandemia da Covid-19 numa perspectiva comparada. Rev. Bras. Educ. Comp., Campinas, 2 (1), 16.

Costa, K. A. S. (2021). Ead, ensino híbrido e ensino remoto emergencial: perspectivas metodológicas. <https://reitoria.ifpr.edu.br/wpcontent/uploads/ 2020/09/EaD-Ensino-Hibrido-e-Ensino-Didatico-Emergencial.pdf>. 
Faria, M. H. D., Francisca, J. P. P., Matheus L. S., Daniela, M. V. P., \& Samara, C. M. S. (2020). Relato de Aluno de Odontologia no Enfrentamento á COVID19. Cadernos Esp Ceará., 14 ( 1), 118-123.

Frison, L. M. B. (2016). Monitoria: uma modalidade de ensino que potencializa a aprendizagem colaborativa e autorregulada. Proposições, 27 (1), $133-153$.

Government Of United Kingdom (2020). Education and childcare: Part of coronavirus. https://www.gov.uk/coronavirus/education-and-childcare.

Hodges, C., Moore, S., Lockee, B., Trust, T., \& Bond, A. (2020). The difference between emergency remote teaching and online learning https://er.educause.edu/articles/2020/3/the-difference-between-emergency-remote-teaching-and-online-learning.

Lins, L. F., Ferreira, L. M. C., Ferraz, L. V., \& Carvalho, S. S. G. (2009). A importância da monitoria na formação acadêmica do monitor. IX Jornada de Ensino, Pesquisa e Extensão.

Matoso, L. M. L. (2014). A Importância Da Monitoria Na Formação Acadêmica Do Monitor: Um Relato De Experiência. Revista Científica da Escola da Saúde, 3 ( 2), 77-83.

Motiejünaitê-Schulmeister, A. \& Crosier, D. (2020). How is Covid-19 affecting schools in Europe? https://eacea.ec.europa.eu/nationalpolicies/eury dice/content/how-covid-19-affecting-schools-europe_en.

Oliveira, M. B. D. E., Silva, L. C. T., Canazaro, J. V., Carvalhido, M. L. L., Souza, R. R. C. D., Neto, J. B., Rangel, D. P., \& Pelegrini, J. F M. (2021). O Ensino Híbrido No Brasil Após Pandemia Do Covid19 / Hybrid Teaching in Brazil After Covid-19 Pandemic. Brazilian Journal of Development, 7 (1), $918-932$.

Oliveira, G. C., Souza, F. P., \& Silva, E. N. (2019). Papel da monitoria na formação acadêmica: um relato de experiência. Revista de Pesquisa Interdisciplinar, 2 (2), 924-926.

Pinto, F. (2021). Mais de mil mortes diárias de Covid-19 - o novo normal? https://portal.fiocruz.br/noticia/mais-de-mil-mortes-diarias-de-covid-19-o-novonormal.

Prieto, D., Tricio J., Cáceres F., Param F., Meléndez C., Vásquez P., \& Prada P. (2020). Academics’ and students’ experiences in a chilean dental school during the Covid-19 pandemic: A qualitative study. European Journal of Dental Education,00, 1-9.

Ruiz, C., \& Dávila, A. (2016). Proposta de boas práticas de educação virtual no contexto universitário. Revista de Educación a Distancia (RED), 49, 1-21.

Spinelli, A \& Pellino, G (2020). COVID-19 pandemic: Perspectives on an Unfolding Crisis. British journal of surgery, 107 (7), $785-787$.

The World Bank (2020). How countries are using edtech (including online learning, radio, television, texting) to support access to remote learning during the COVID-19 pandemic https://www.worldbank.org/en/topic/edutech/brief/how-countries-are-using-edtech-to-support-remote-learning-during-the-covid-19pandemic.

Tokuç, Burcu, Varol, Gamze. (2020). Medical Education in Turkey in the Time of COVID-19. Balkan Medical Journal, 37 (4), $180-181$.

United Nations Educational, Scientific and Cultural Organization (2020). COVID19 educational disruption and response. https://en.unesco.org/themes/education-emergencies/coronavirus-school-closures.

Vicenzi, C. B., Conto, F., Flores, M. E., Rovani, G., Ferraz, S. C. C., \& Marostega, M. G (2016). A monitoria e seu papel no desenvolvimento da formação acadêmica. Rev. Ciênc. Ex, 12 (3), 88-94.

World Health Organization (2020). Global research on Coronavirus disease (COVID-19). https://www.who.int/emergencies/diseases/novel-coronavirus2019/global-research-on-novel-coronavirus-2019-ncov. 